Bibliotecas. Vol 39, № 2, julio - diciembre, 2021. EISSN: 1659-3286

URL: http://www.revistas.una.ac.cr/index.php/bibliotecas/index

DOI: http://dx.doi.org/10.15359/rb.39-2.3

Licencia: Creative Commons (BY-NC-SA) 4.0 Internacional

\title{
Revistas ambientales latinoamericanas en Scopus y WoS en 2019:
} relación con indicadores ambientales

\author{
Latin American Environmental Journals in Scopus and WoS in 2019: Relationship with \\ Environmental Indicators
}

Tomás Darío Marín Velásquez. Universidad de Oriente, Venezuela ORCID: https://orcid.org/0000-0002-3334-5895

\author{
Dany Day Josefina Arriojas Tocuyo. \\ Petróleos de Venezuela, Venezuela \\ ORCID: https://orcid.org/0000-0002-8192-9641
}

Recibido: 04 de febrero de 2021

Aceptado: 23 de marzo de 2021

Publicado: 21 de junio de 2021

\begin{abstract}
Resumen
La investigación tuvo como objetivo analizar las revistas de ciencias ambientales de ALyC indexadas en Scopus y WoS en 2019, así como su relación con ciertos indicadores ambientales. Se realizó con enfoque cuantitativo y nivel explicativo, a partir de datos obtenidos de los portales oficiales de Scopus y WoS. Los datos bibliométricos fueron los países que poseen revistas indexadas, el Número de Revistas, el Factor de Impacto, Número de Documentos Publicados, Número de Citas, Número de Autocitas, Relación de Citas por Documentos, Relación de Citas Externas por Documento, Porcentaje de Colaboración Internacional y Cuartil de ubicación de cada revista. Los indicadores ambientales fueron tomados del portal oficial del Banco Mundial y los mismos fueron el Uso de la Energía, Áreas Protegidas, Contaminación por PM2.5, Emisiones de $\mathrm{CO}_{2}$ y Rentas Totales por Recursos Naturales. Los análisis estadísticos se basaron en técnicas descriptivas y multivariadas, como análisis de conglomerados, correlaciones canónicas y regresión multivariada, todos con un nivel de confianza de 95\%. Se obtuvieron 48 revistas Scopus y 29 WoS, con Brasil, Chile, Colombia y México como los países con mayor presencia de revistas. En los indicadores ambientales destacan Brasil y Chile. Las variables bibliométricas que se relacionan significativamente con indicadores ambientales fueron Número de Revistas y Autocitas. El indicador ambiental que se relaciona positivamente con las variables bibliométricas fue las Áreas Protegidas y de forma negativa la Contaminación por PM2.5.
\end{abstract}

Palabras clave: bibliometría, indicadores bibliométricos, Latinoamérica, revistas ambientales, visibilidad. 


\begin{abstract}
The research aimed to analyze the ALyC journals indexed in Scopus and WoS around environmental sciences in 2019, as well as their relationship with certain environmental indicators. It was carried out with a quantitative approach and an explanatory level, based on data obtained from the official Scopus and WoS portals. The bibliometric data were the countries that have indexed journals, the Number of Journals, the Impact Factor, Number of Published Documents, Number of Citations, Number of Self-Quotations, Ratio of Citations per Document, Ratio of External Citations per Document, Percentage of International Collaboration and Quartile of location of each journal. The environmental indicators were taken from the World Bank's official website and were Energy Use, Protected Areas, PM2.5 Pollution, $\mathrm{CO}_{2}$ Emissions and Total Natural Resource Income. The statistical analyses were based on descriptive and multivariate techniques, such as cluster analysis, canonical correlations, and multivariate regression, all with a 95\% confidence level. There were obtained 48 Scopus journals and 29 WoS, with Brazil, Chile, Colombia and Mexico as the countries with greater presence of magazines in the region. In the environmental indicators, Brazil and Chile stand out. The bibliometric variables that are significantly related to environmental indicators were the Number of Journals and Selfcitations. The environmental indicator that is positively related to bibliometric variables was Protected Areas and negatively related to PM2.5 pollutions.
\end{abstract}

Keywords: bibliometrics, indicators, Latin America, environmental journals, visibility.

\title{
I. Introducción
}

La producción y difusión científica es fundamental para el desarrollo de la ciencia y por ende de los países. En el caso de América Latina y el Caribe (ALyC), su producción científica es considerablemente baja en todas las áreas, en comparación con otras regiones como Europa, Asia y Norteamérica (Ibáñez, 2017). Lo anterior, se debe en general a que es una región con gran desequilibrio político, social y económico; problemas que se presentan en la mayoría de los países de la región, que hacen que tenga baja producción científica a nivel mundial (Santa y Herrero 2010; Guerrero-Casado, 2017).

A pesar de ser ALyC una región con gran potencial desde el punto de vista agroforestal, turístico y de recursos naturales, la cantidad de sus revistas en el área de ciencias ambientales y en general en todas las áreas, deriva de la escasa calidad de las revistas de la región que ha dificultado su inclusión en bases de datos internacionales, lo que ha llevado a una escasa presencia de revistas latinoamericanas en las bases de datos del Web of Science (WoS) y Scopus (Miguel, 2011). Esto a pesar de que la cantidad de revistas de ALyC y su producción ha aumentado en los últimos años, como el caso particular de las revistas en Scopus, las cuales pasaron de 802 revistas en 2015 a 909 en 2019, lo que 
representa un aumento del $13,3 \%$ y el número de documentos publicados también mostró un aumento de $25,8 \%$ en el mismo lapso, lo que fue muy superior al 1,7\% de aumento de América del Norte, aún sigue siendo una producción baja, ya que el número de revistas sigue siendo insuficiente.

A lo largo de los años, se han desarrollado investigaciones bibliométricas donde se ha analizado la producción científica latinoamericana en forma general (Montilla, 2012; León, Socorro, Cáceres y Pérez, 2020) y en áreas específicas como las ciencias agropecuarias (Guerrero-Casado, 2017), en ciencias naturales (Crespo-Gascón, Tortosa y GuerreroCasado, 2019) y en el área de ciencias de la salud (Ortega-Loubon et al., 2013; Luna-Solís, 2015).

El número de revistas y su producción, es dependiente de las políticas propias de cada país, así como de la importancia de la ciencia en la región. Un área que ha estado en auge en los últimos años es el de las ciencias ambientales, a pesar de ello, el tema ambiental, aun cuando es parte de la agenda de ciencia y tecnología en la mayoría de los países, todavía se continúa tratando como un tema sectorizado y de poco interés público y privado (Celaya et al., 2017). Asimismo, Casas et al. (2017) considera que se presentan iniciativas individuales de investigadores y ciudadanos preocupados por los problemas ambientales que son todas de gran valor, pero debe haber compromiso por parte de las instituciones para dar atención colectiva a problemas que rebasan las posibilidades de los individuos. Este es un gran reto, que implica el trabajo conjunto de instituciones gubernamentales, no gubernamentales, organizaciones sociales, e instituciones académicas para lograr mejores resultados en temas ambientales.

Con base en lo anterior se planteó la presente investigación que tuvo como objetivo analizar las revistas de ALyC indexadas en las bases de datos Scopus y WoS en el área de ciencias ambientales para el año 2019, su impacto y visibilidad, así como su relación con indicadores ambientales, para entender cómo estos indicadores influyen en la producción y difusión de la investigación en el área temática. 


\section{Materiales y métodos}

El número de revistas Scopus se obtuvieron del portal Scimago Journal and Country Rank (https://www.scimagojr.com), que es una herramienta creada por SCImago Research Group, en el que se muestran indicadores científicos de revistas incluidas en Scopus y los países a los que pertenecen. Se filtró la información por las revistas incluidas en el área Environmental Science, por cada país de la región de América Latina para el año 2019. De igual manera se extrajeron datos de Factor de Impacto $(S J R)$, número de documentos publicados (Documentos), número de citas (Citas), número de autocitas (Autocitas), relación de citas por documentos (Citas/Documentos), relación de citas externas por documento (Citas Externas), porcentaje de colaboración internacional (\%Colab. Inter.) y cuartil de ubicación de cada revista.

El número de revistas WoS se obtuvo del portal Web of Science Master Journal List (https://mjl.clarivate.com). Se filtró por las áreas de Environmental Science y Environmental Studies para cada uno de los países de ALyC. Asimismo, se extrajo información sobre la ubicación de cada revista dentro del Web of Science Coverage (Core Collection y Current Contents) y el Factor de Impacto (JCR).

Los indicadores ambientales para cada uno de los países con revistas indexadas en Scopus y WoS en el área de ciencias ambientales, se obtuvieron del portal oficial de World Bank (World Bank Group, 2020) siendo las mismas, el Uso de la Energía (kg de equivalente de petróleo per cápita), Áreas Protegidas (Terrestres y Marinas \% del total de la superficie territorial), Contaminación por PM2.5 (\% del total de población expuesta), Emisiones de $\mathrm{CO}_{2}$ (Toneladas métricas per cápita) y Rentas Totales por Recursos Naturales (\%PIB).

Los datos fueron procesados mediante tablas y gráficas utilizando hojas de cálculo de Libre Office Calc. Los análisis estadísticos se basaron en técnicas multivariantes, tanto para la estadística descriptiva mediante análisis de conglomerados (De La Fuente, 2011) así como para estudiar las posibles relaciones entre las variables bibliométricas y los indicadores ambientales, mediante correlaciones canónicas (Cuadras, 2014). De igual forma se realizó la comparación entre el número de revistas Scopus y WoS mediante la prueba de Kolmogorov-Smirnov para comparación de distribuciones (Amat, 2020). Todos los análisis estadísticos se realizaron mediante el paquete estadístico Statgraphics Centurion XVII. 


\section{Resultados}

Luego de obtener el número de revistas del área de ciencias ambientales indexadas en Scopus y WoS, se elaboró la gráfica que se muestra en la Figura 1. En total se obtuvieron 48 revistas Scopus y 29 revistas WoS, donde se observa que en ambas bases de datos, Brasil posee la mayor cantidad de revistas indexadas, con 29 en Scopus $(60,42 \%)$ y 17 en WoS (58,62\%). En segundo lugar, respecto a las revistas Scopus, se encuentra México con $7(14,58 \%)$ y en WoS Chile con $4(13,79 \%)$. El tercer país con el mayor número de revistas Scopus de ciencias ambientales es Colombia con $6(12,5 \%)$ y en el caso de WoS este lugar lo comparten Colombia, Ecuador y México con $2(6,90 \%)$ cada uno. Se realizó un análisis de igualdad de distribuciones a partir de la prueba de Kolmogorov-Smirnov para determinar si las tendencias de las cantidades de revistas son equivalentes y los resultados se muestran en la Figura 2.

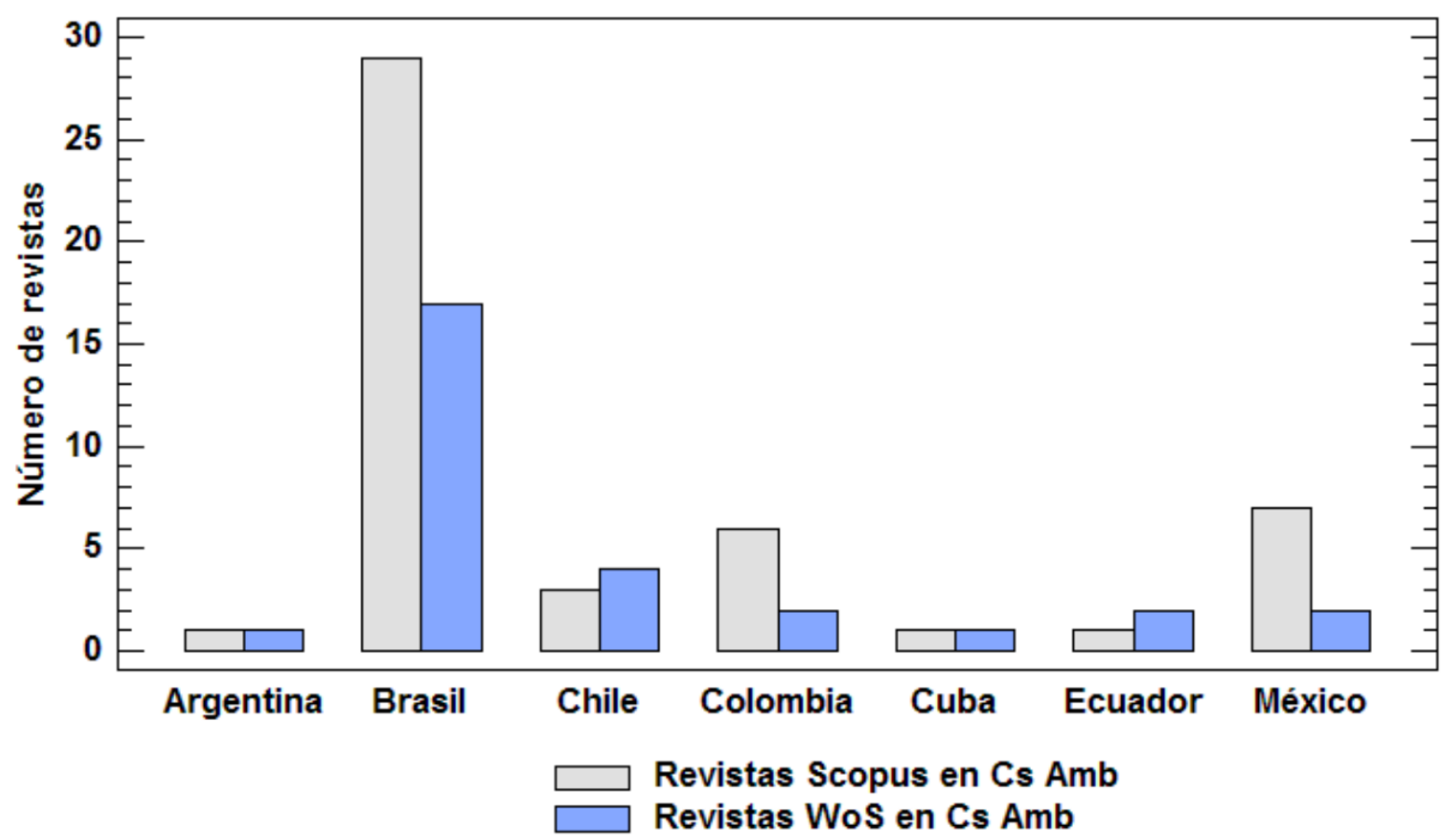

Figura 1. Número de revistas del área de ciencias ambientales indexadas en Scopus y WoS, año 2019.

Fuente: Elaboración propia de las personas autoras, 2021. 
Revistas Scopus de Cs Amb

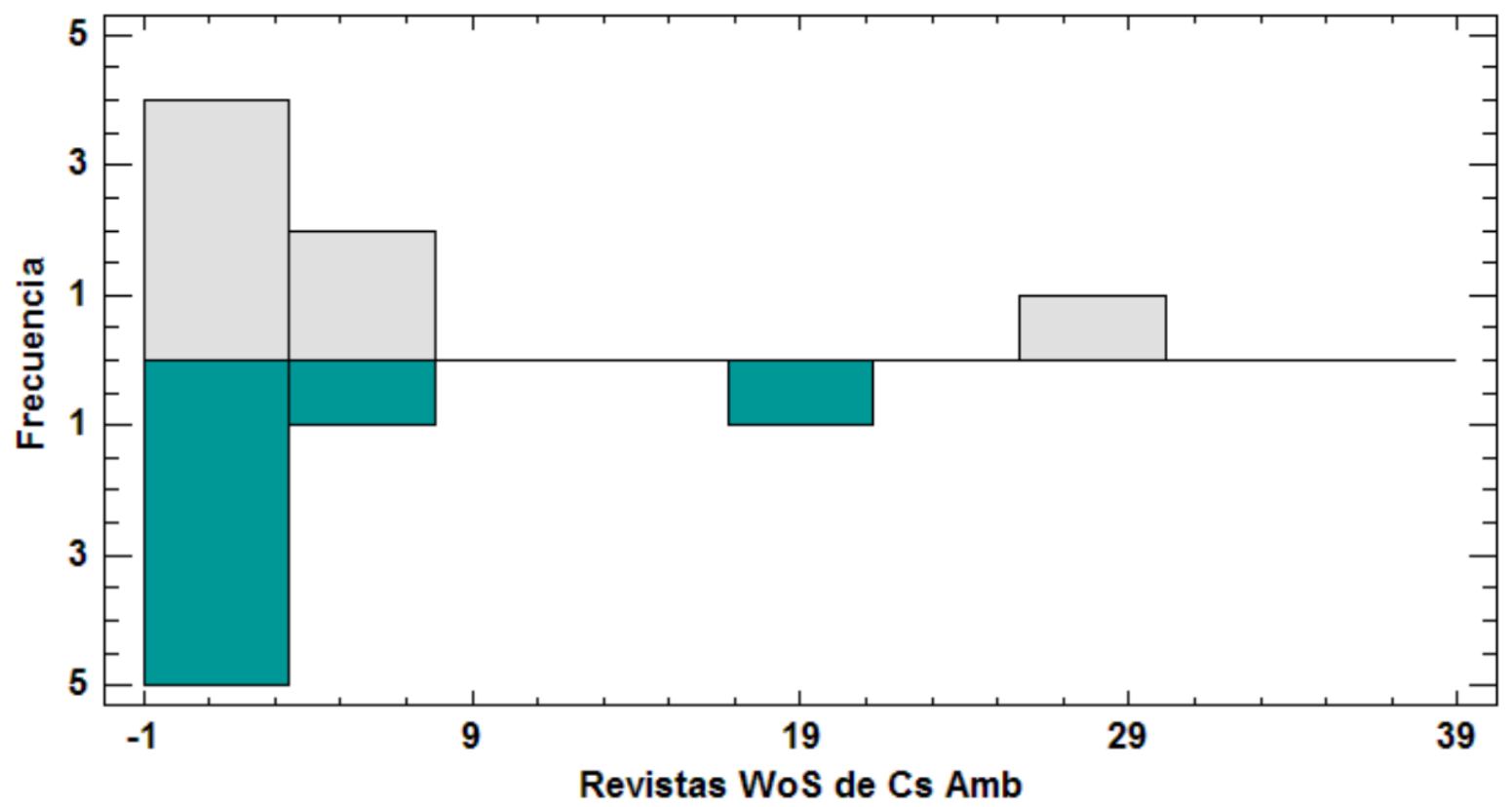

Figura 2. Gráfica de distribución comparada del número de revistas Scopus y WoS en ciencias ambientales para el año 2019.

Fuente: Elaboración propia de las personas autoras, 2021.

Como se observa en la figura 2, la distribución comparada de las revistas Scopus y WoS respecto a los países es similar, lo que se corrobora con el estadístico de KolmogorovSmirnov, el cual fue K-S bilateral $=0,53$ con un valor- $p=0,9375$ que al ser mayor que $\alpha=$ 0,05 indica que no existe diferencia significativa entre las dos distribuciones, por lo que la tendencia del número de revistas en ambas indexadoras es el mismo. Otro punto importante es que se encuentran ocho revistas con indexación en ambas bases de datos lo que representa un 15,09\% de solapamiento calculado a partir de la ecuación de Gluck (Escalona, Lagar y Pulgarín, 2010).

Las variables bibliométricas como número de Documentos, Citas y Autocitas, están relacionadas con el número de revistas, ya que es evidente que mientras más revistas tiene un país, mayores serán estas variables, lo que puede observarse en la Figura 3, donde destacan Brasil y México por tener la mayor cantidad de revistas en Scopus. 


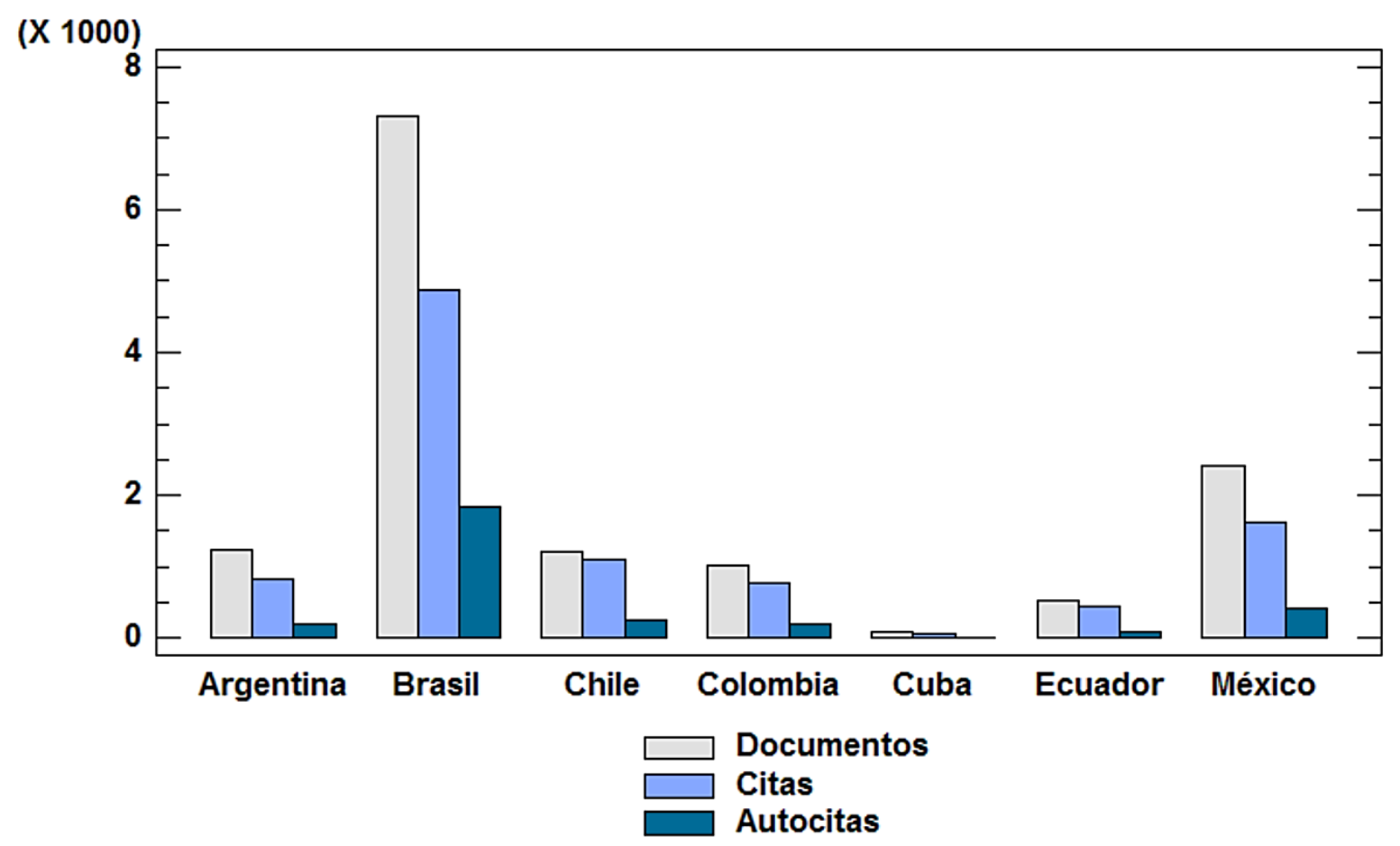

Figura 3. Distribución de las variables Documentos, Citas y Autocitas de las revistas de ciencias ambientales en los Países de ALyC, año 2019.

Fuente: Elaboración propia de las personas autoras, 2021.

Las variables bibliométricas, como $S J R$, Citas/Documentos y Citas externas, presentan la distribución que se observa en la Figura 4. Respecto al factor de impacto (SJR), Argentina, Brasil y Chile presentan valores similares $(0,277 ; 0,269$ y 0,264 respectivamente) con Argentina como el país con las revistas de ciencias ambientales con mayor impacto en ALyC, a este grupo le siguen México, Colombia y Ecuador.

En las Citas/Documentos destacan Chile con 0,92 seguido por Ecuador con 0,85 y Colombia con 0,75 como los tres países que recibieron mayor cantidad de citas en relación con los documentos publicados para el 2019. Luego Argentina, Brasil y México se ubican con valores similares de 0,$67 ; 0,67$ y 0,66 respectivamente, dejando a Cuba con 0,55 al final de la tabla. Estos datos indican que los documentos de las revistas chilenas fueron más citados en el año 2019, con una mención especial a Ecuador que aun cuando tiene una sola revista en Scopus, el número de Citas/Documentos publicados por dicha revista superaron la mayoría de los países. (Figura 4). 


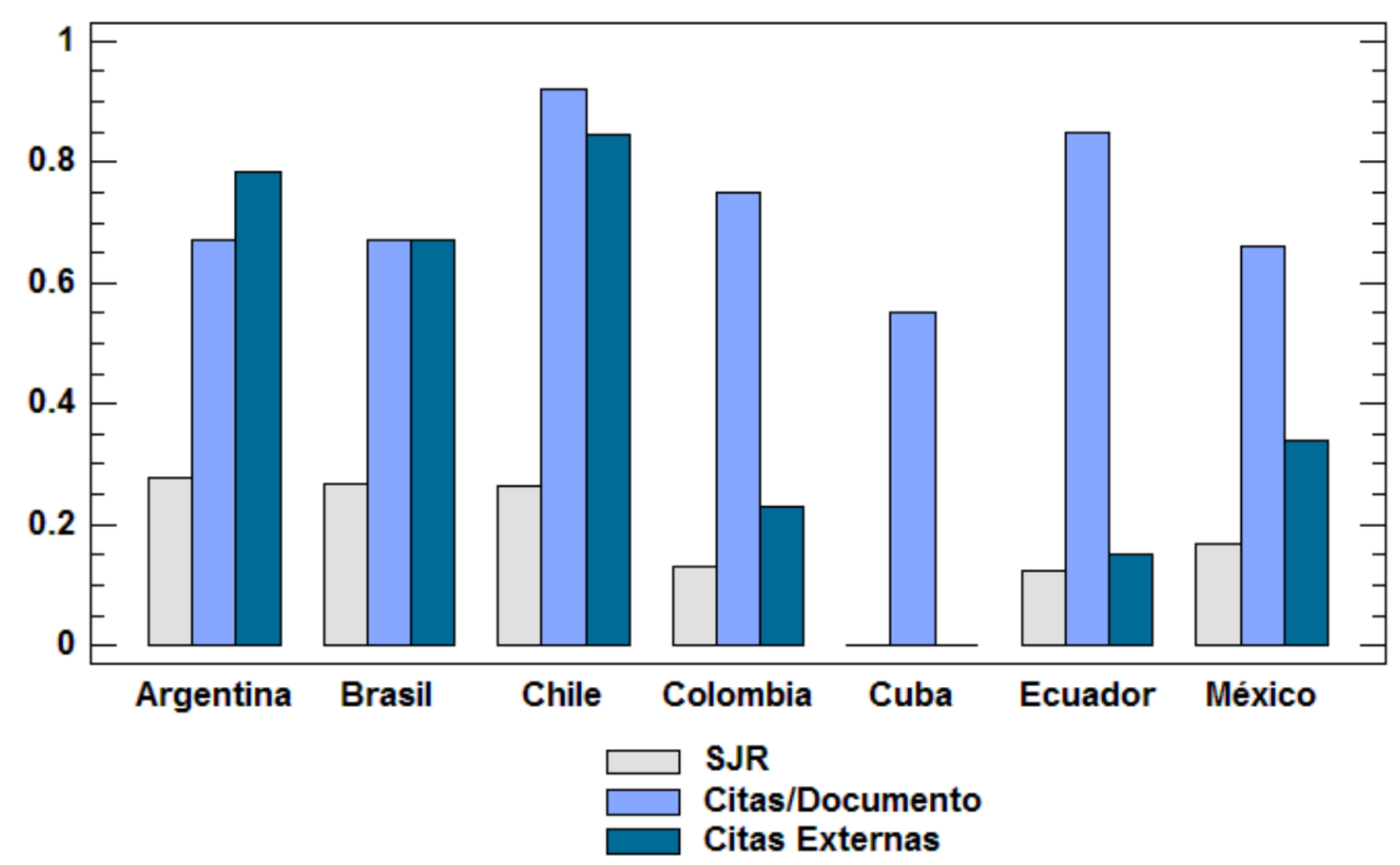

Figura 4. Distribución de las variables SJR, Citas/Documentos y Citas Externas de las revistas de ciencias ambientales en los Países de ALyC, año 2019.

Fuente: Elaboración propia de las personas autoras, 2021.

El número de citas externas por documentos favorece a las revistas chilenas con 0,847 , seguidas por las revistas argentinas con 0,784 y las brasileñas con 0,671 , les siguen las revistas mexicanas, colombianas y ecuatoriana. Las revistas chilenas también se destacaron respecto a las citas externas.

El número de colaboración internacional se muestra gráficamente en la Figura 5 favoreciendo a la revista ecuatoriana con un porcentaje de $28,57 \%$, seguida por las revistas de México con $16,79 \%$ y las argentinas con $14,63 \%$.

La distribución de las revistas de ciencias ambientales indexadas en Scopus, respecto al cuartil donde se ubica de acuerdo con $S J R$, se muestra en la Figura 6 . Se observa que la mayor cantidad de revistas se encuentran clasificadas como Q3 y Q4 con 22 y 19 respectivamente, tres revistas se clasifican como Q2 y una como Q1. 
Bibliotecas. Vol. 39, № 2, julio - diciembre, 2021. EISSN: 1659-3286

URL: http://www.revistas.una.ac.cr/index.php/bibliotecas/index

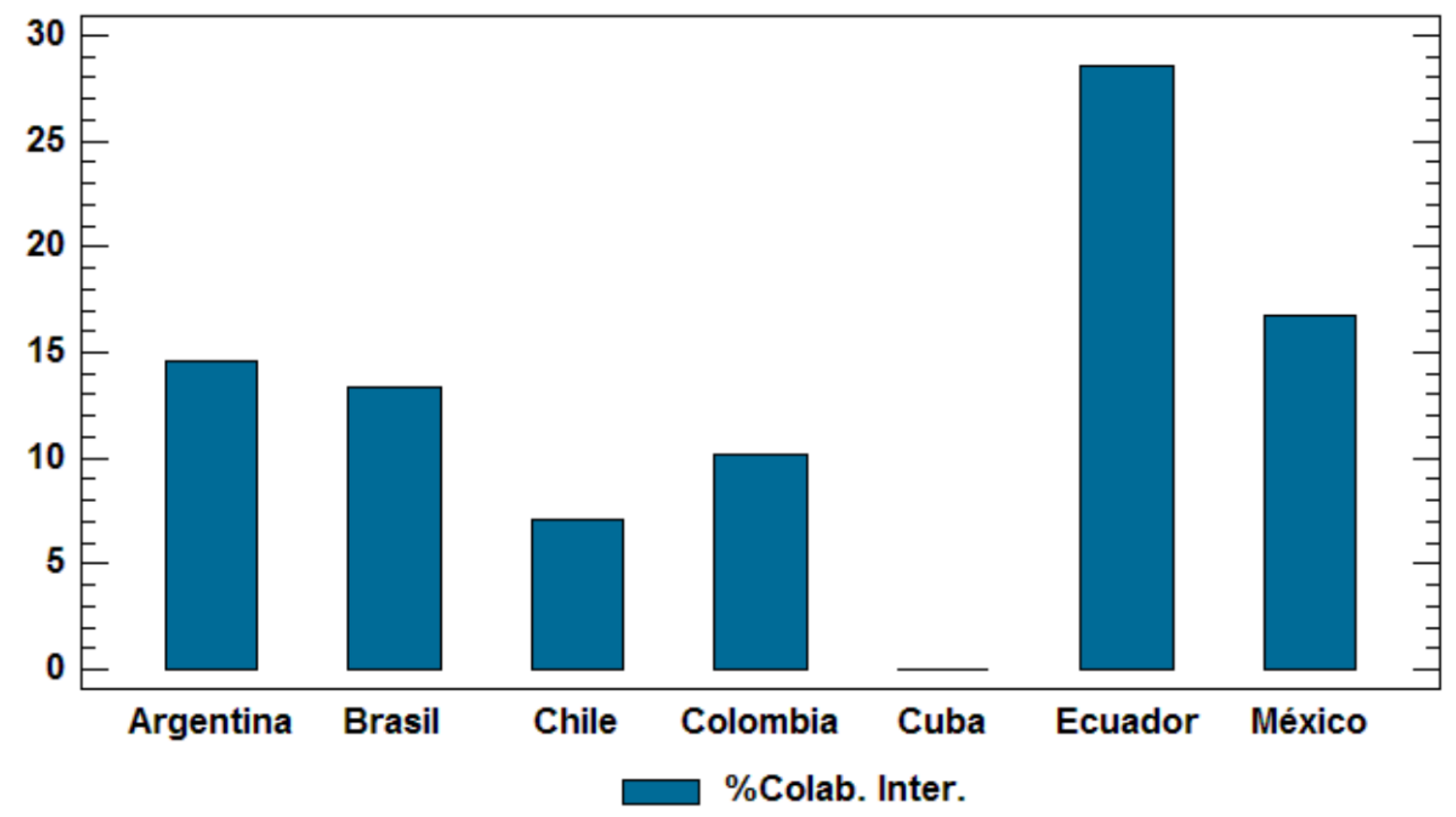

Figura 5. Distribución de la variable \%Colab. Inter. de las revistas de ciencias ambientales en los Países de ALyC, año 2019.

Fuente: Elaboración propia de las personas autoras, 2021.

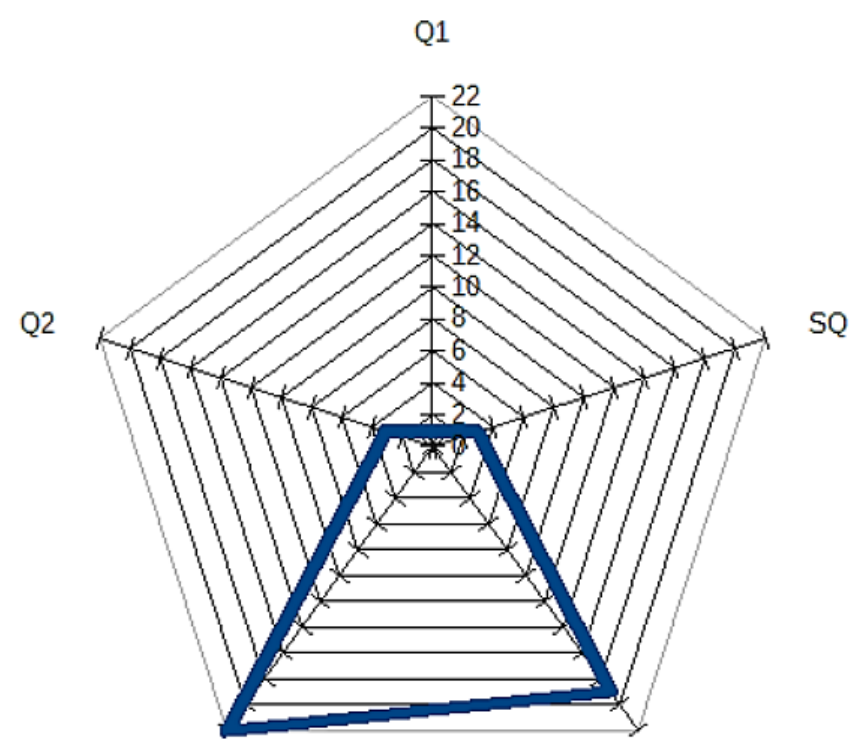

Q3

Q4

Figura 6. Distribución de las revistas de ciencias ambientales indexadas en Scopus de acuerdo con el cuartil.

Fuente: Elaboración propia de las personas autoras, 2021. 
Tres revistas no aparecen ubicadas en los cuartiles, debido a que son de reciente ingreso a la indexadora y no han sido evaluadas todavía. Se observa, entonces que la mayoría de las revistas de ciencias ambientales Scopus de ALyC (85,42\%) se ubican en los cuartiles bajos. La única revista ubicada en el cuartil superior (Q1) es brasileña y las del segundo cuartil (Q2) son dos brasileñas y una chilena, lo que ubica a Brasil con la revista de mayor impacto en esta área, de la región. El análisis de las revistas WoS mostró que la mayoría (13) se encuentran indexadas dentro de la colección de revistas emergentes (ESCl) como se muestra en la Figura 7.

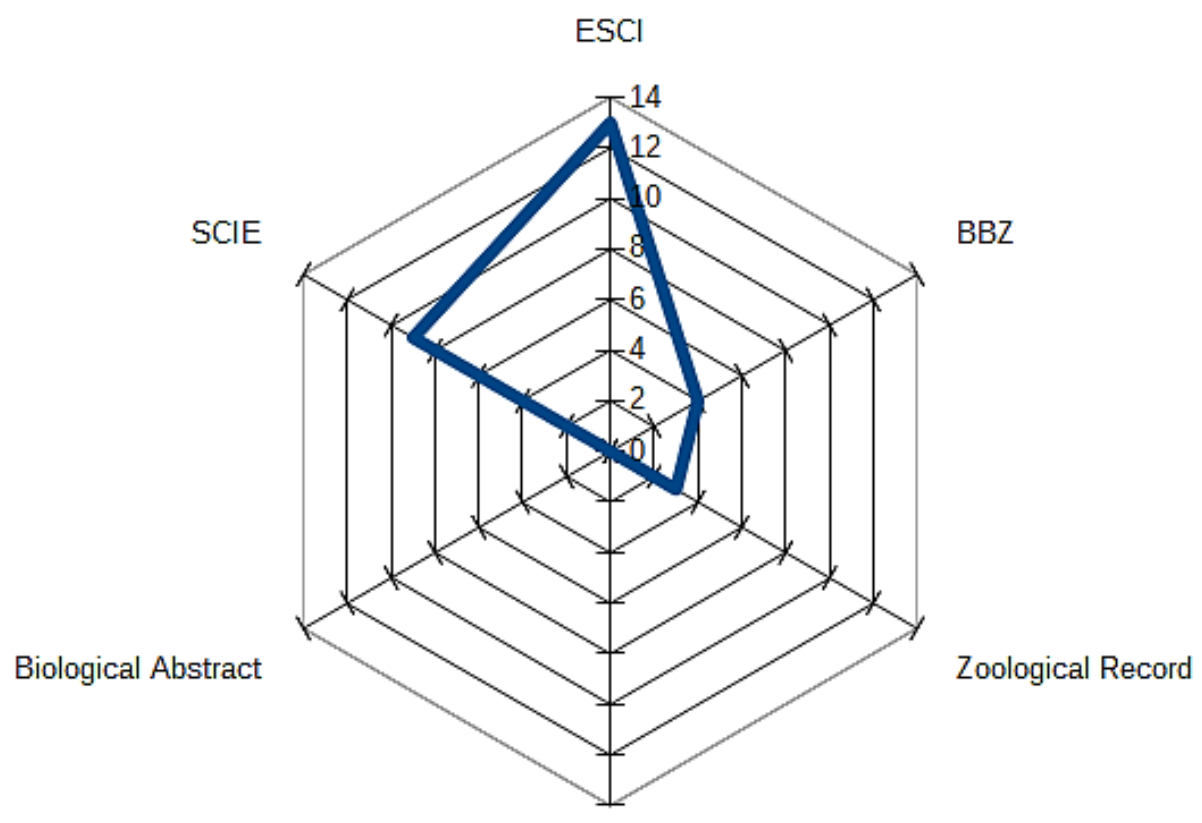

BIOSIS Previews

Figura 7. Distribución de las revistas de ciencias ambientales de ALyC indexadas en WoS para el año 2019.

Fuente: Elaboración propia de las personas autoras, 2021.

También se observa en la Figura 7 que nueve revistas se encuentran dentro de la colección Science Citation Index Expanded (SCIE) las cuales representan las de mayor impacto por estar indexadas dentro de uno de los núcleos principales de WoS. Por otro lado, tres revistas se encuentran ubicadas en el índice Zoological Record y cuatro tienen indexación conjunta o solapada en los índices Biological Abstract, BIOSIS Previews y Zoological Record (BBZ). Los países con revistas incluidas en el SCIE fueron de Brasil, Chile y México (4, 3 y 2 respectivamente) por lo tanto son los únicos que presentan factor de impacto $J C R$, siendo el orden Brasil $(1,293)$, Chile $(1,07)$ y México $(0,742)$. 
Para observar la clasificación y agrupamiento de las revistas de ciencias ambientales indexadas en Scopus de acuerdo con el país de origen en función a las variables bibliométricas, se realizó un análisis de clústeres o conglomerados, cuyo resultado se muestra en el dendograma de la Figura 8.

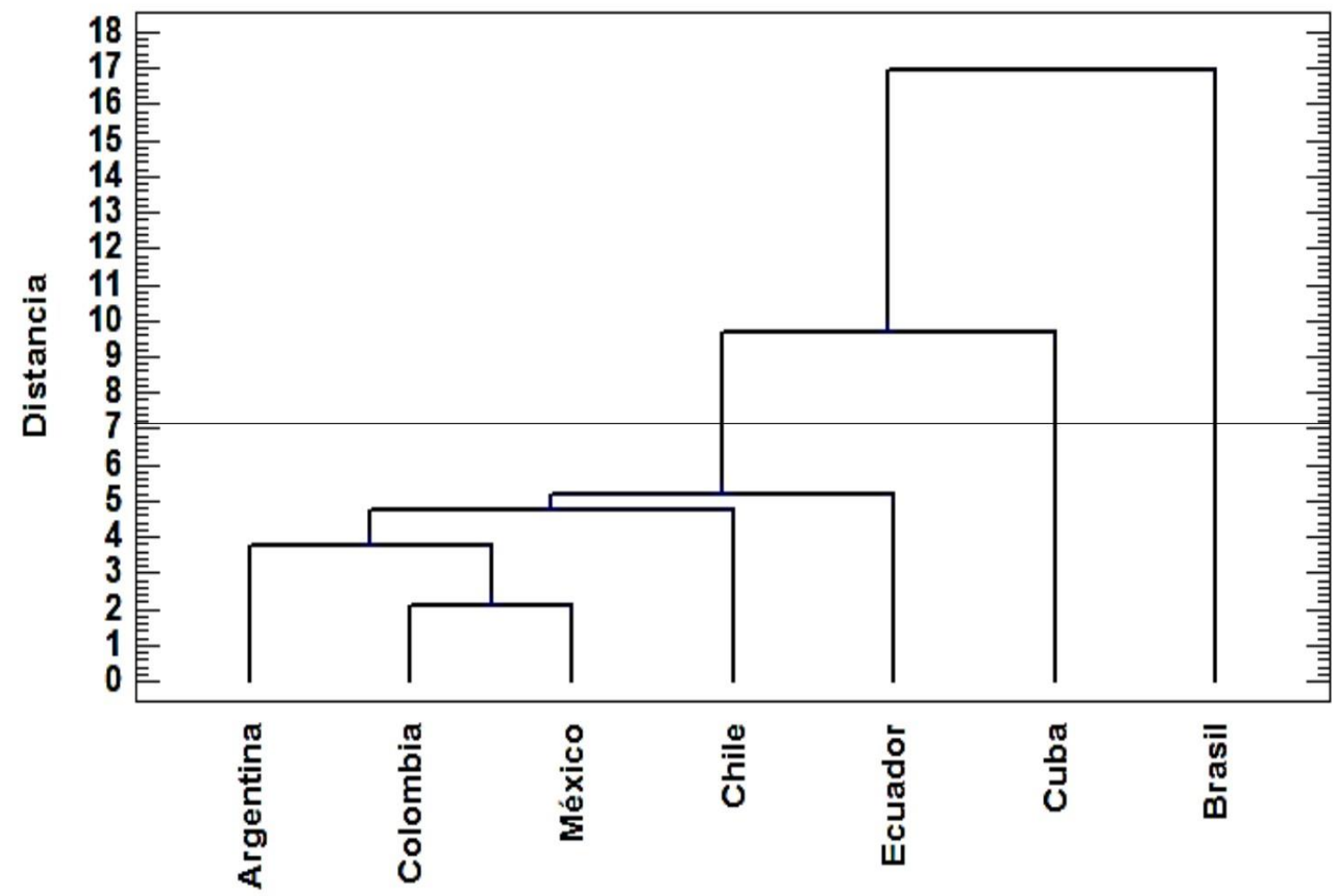

Figura 8. Dendograma de agrupamiento de las revistas por país de acuerdo con las variables bibliométricas.

Fuente: Statgraphics Centurion XVII.

En el dendograma se observa que al colocar como línea de corte la distancia promedio que fue de 7,06 se obtienen tres conglomerados, el primero donde se agrupan Argentina, Colombia, México, Chile y Ecuador, el segundo donde se encuentra solo Cuba y por último Brasil. Lo anterior indica que, a pesar de las diferencias entre las variables bibliométricas que se observan en las revistas, el comportamiento global es similar entre los países de la región, donde solo se destacan las revistas brasileñas, por ser mayoría y la única revista cubana, la cual se separa del resto, porque al ser de reciente ingreso a Scopus no presenta valores en algunas de las métricas. 
Respecto a los indicadores ambientales, los países se agrupan tal como se observan en el dendograma de la Figura 9, con una línea de corte que representa la distancia media de agrupamiento, que fue de 4,8 .

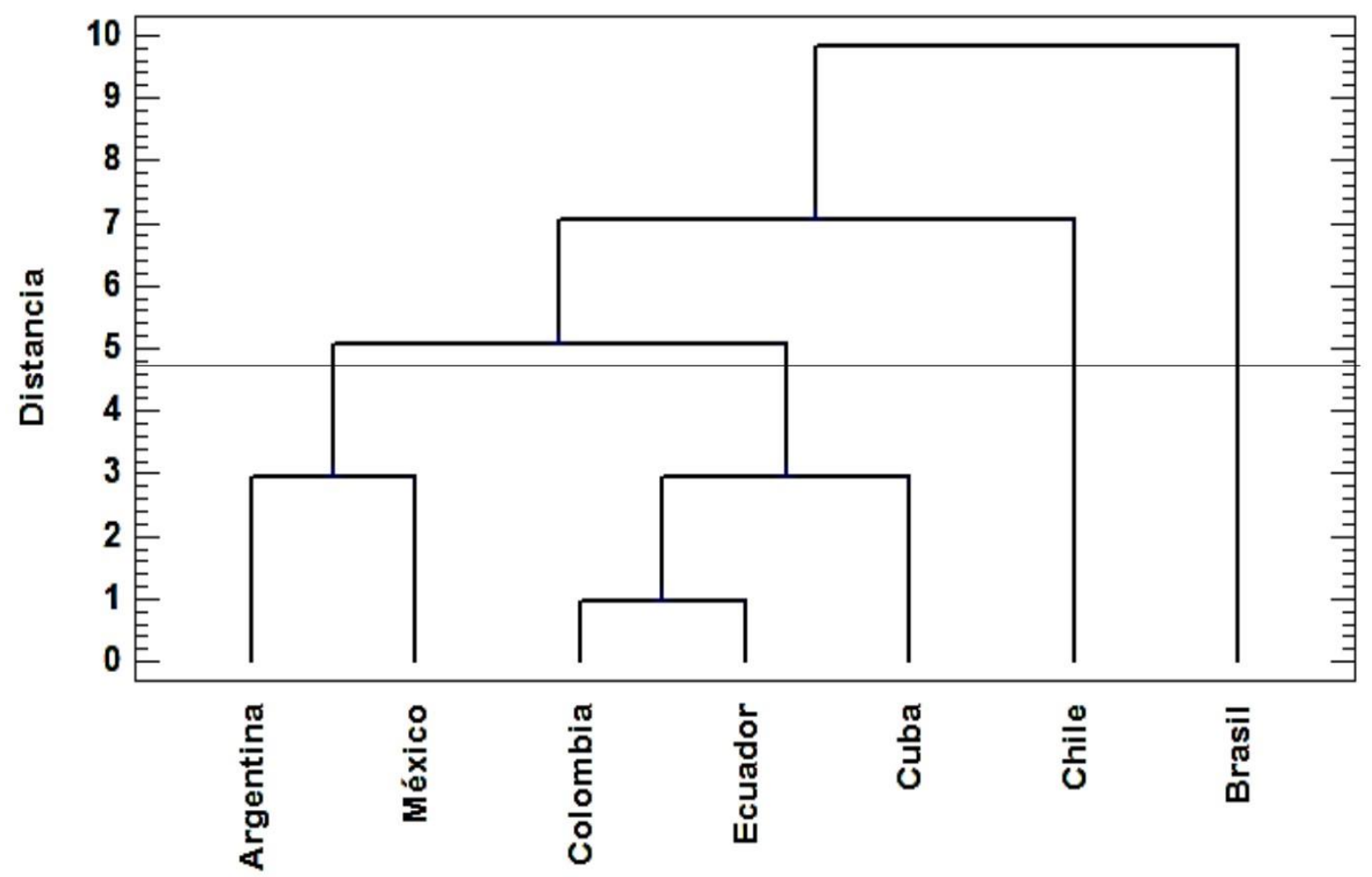

Figura 9. Dendograma de agrupamiento de los países de acuerdo con los indicadores ambientales.

Fuente: Statgraphics Centurion XVII.

Los países latinoamericanos con revistas del área de ciencias ambientales indexadas en Scopus y WoS se agrupan de acuerdo con sus indicadores ambientales en cuatro clústeres o conglomerados en los que internamente no existen diferencias significativas. En el primer clúster se encuentran Argentina y México, en el segundo se agrupan Colombia, Ecuador y Cuba, por su parte Chile y Brasil se clasifican de forma independiente, es decir que los indicadores ambientales de estos dos países no presentan similitud con los demás países, por lo que se ubican cada uno en un clúster único.

La aplicación de la técnica estadística de correlación canónica arrojó los resultados que se muestran en la Figura 10, donde se observa que las variables bibliométricas cuya relación 
con los indicadores ambientales es significativa (Valor-p $<0,05$ ) son las Revistas, Documentos, Citas, Autocitas y Citas/Documentos.

\begin{tabular}{|c|c|c|c|c|c|c|}
\hline & & eficie & te de C & rrelaci & & \multirow{2}{*}{$\begin{array}{c}\text { Valor-p } \\
0.000\end{array}$} \\
\hline Revistas & 0.02 & 0.67 & -0.96 & -0.31 & -0.15 & \\
\hline SJR & 0.73 & 0.55 & -0.47 & 0.57 & 0.32 & 0.230 \\
\hline Documentos & 0.18 & 0.67 & -0.94 & -0.14 & -0.15 & 0.010 \\
\hline Citas & 0.21 & 0.72 & -0.94 & -0.12 & -0.08 & 0.010 \\
\hline Autocitas & 0.15 & 0.68 & -0.96 & -0.19 & -0.12 & 0.040 \\
\hline Citas/Documento & 0.12 & 0.49 & 0.15 & 0.22 & 0.95 & 0.030 \\
\hline$\%$ Autocitas & 0.23 & 0.75 & -0.77 & 0.01 & 0.15 & 0.140 \\
\hline Citas Externas & 0.85 & 0.50 & -0.37 & 0.68 & 0.35 & 0.200 \\
\hline$\%$ Colab. Inter. & 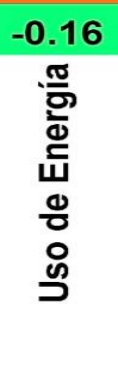 & 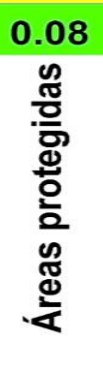 & 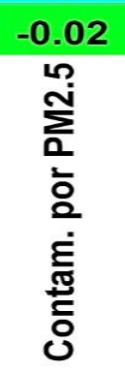 & 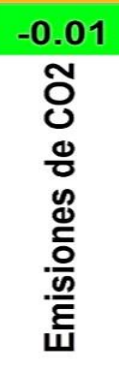 & 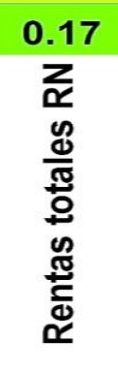 & 0.220 \\
\hline
\end{tabular}

Figura 10. Representación gráfica de los coeficientes de correlaciones canónicas y su nivel de significancia entre las variables bibliométricas y los indicadores ambientales.

Fuente: Statgraphics Centurion XVII.

El número de revistas tiene una relación alta y positiva con las áreas protegidas y de moderada a muy alta negativa con Emisiones de $\mathrm{CO}_{2}$ y Contaminación por PM2.5. Lo anterior sugiere que los países con mayor cantidad de revistas tienden a ser las que poseen mayores cantidades de áreas protegidas y presentan menores niveles de contaminación del aire. El resto de las variables bibliométricas con relaciones significativas siguen el mismo patrón que el número de revistas, con la excepción de las Citas/Documentos que muestra relación positiva muy alta con las rentas totales por recursos naturales y su relación con los indicadores de contaminación ambiental son bajos.

A partir de los resultados de correlación se realizó un análisis de regresión lineal multivariada con las variables bibliométricas que mostraron relaciones significativas con los indicadores bibliométricos y los resultados se muestran en el Cuadro 1. 


\section{Cuadro 1}

\section{Resultados del análisis de regresión lineal multivariada de las variables bibliométricas y los indicadores ambientales}

\begin{tabular}{|c|c|c|c|c|}
\hline Variable & $\mathbf{R}^{\mathbf{2}}$ & $\mathbf{R}^{\mathbf{2}}$ Ajustada & Variables Incluidas & Valor-p \\
\hline Revistas Scopus & 99,95 & 99,85 & ABCE & 0,0010 \\
\hline Revistas WoS & 97,75 & 96,63 & BC & 0,0005 \\
\hline Documentos & 99,65 & 97,89 & ABCDE & 0,0990 \\
\hline Citas & 99,74 & 98,47 & ABCDE & 0,0846 \\
\hline Autocitas & 98,50 & 96,99 & BCE & 0,0031 \\
\hline Citas/Documentos & 99,30 & 95,80 & ABCDE & 0,1396 \\
\hline
\end{tabular}
A: Uso de Energía,
B: Áreas protegidas,
C: Contam. por PM2.5,
D: Emisiones de $\mathrm{CO}_{2}$,
E: Rentas totales RN.
Fuente: Elaboración propia de las personas autoras, 2021.

Se observa en el Cuadro 1 que los indicadores ambientales predicen la variabilidad de las variables bibliométricas en rangos entre 95,80 a $99,85 \%$. En el caso de las variables Documentos, Citas y Citas/Documentos, todos los indicadores ambientales son regresoras, por su parte las Revistas Scopus son predichas por el Uso de Energía, Áreas protegidas, Contam. por PM2.5 y Rentas totales RN. Las Revistas WoS son predichas por los indicadores Áreas protegidas y Contam. por PM2.5. Las Autocitas se relacionan con las variables Áreas protegidas, Contam. por PM2.5 y Rentas totales RN. El Valor-p indica que las variables Documentos, Citas y Citas/Documentos, a pesar de su alto valor de $\mathrm{R}^{2}$, son predichas por modelos de regresión lineal múltiple que no son significativos (Valor-p $>0,05$ ) lo que deja a las variables bibliométricas Revistas y Autocitas como las que tienen un comportamiento que se relaciona y es predicho significativamente por indicadores ambientales. 
Las ecuaciones que rigen el comportamiento de la cantidad de revistas y las autocitas se muestran a continuación:

Revistas Scopus $=56,0699-0,00146279 * A+0,771491 * B-0,575701 * C-1,11029 * E$

Revistas $W o S=51,2318+0,165689 * B-0,526055 * C$

Autocitas $=4000,04+39.9875 * B-41,8977 * C-54,2789 * E$

En todos los casos el principal efecto positivo lo representa la cantidad de áreas protegidas que poseen los países y el principal efecto negativo es el de las Rentas totales por recursos naturales, con un efecto negativo también importante de la cantidad de Contaminación por PM2.5 de los países.

\section{Discusión}

El número de revistas científicas de ciencias ambientales de ALyC indexadas en las bases de datos Scopus y WoS, representan el 5,69 y 2,49\% respectivamente del total de revistas que poseen los países tomados para este estudio. Estos porcentajes son bajos y denotan la poca presencia de revistas ambientales en las dos principales bases de datos de indexación internacional, sobre todo en WoS. Esta escasa presencia de revistas no es exclusiva del área de ciencias ambientales, ya que Rogel-Salazar, Santiago-Bautista y MartínezDomínguez (2017) también reportaron baja cantidad de revistas de ciencias de la comunicación en ambas bases de datos, al igual que Carvajal-Tapia y Carvajal-Rodríguez (2019) al estudiar revistas del área de ciencias de la salud de ALyC.

La tendencia de las revistas de ciencias ambientales de ALyC fue la misma desde el punto de vista estadístico al comparar entre las dos bases de datos, favoreciendo abiertamente a Brasil, detalle que ha sido reportado en otras investigaciones previas con la de Miguel (2011) y Carvajal-Tapia y Carvajal-Rodríguez (2019). Es evidente que Brasil, México y Colombia lideran en cuanto al número de revistas del área indexadas en Scopus y WoS. Brasil se ha consolidado como el país de ALyC con la mayor cantidad de revistas indexadas y la mayor producción científica en todas las áreas, como lo muestra Rogel-Salazar, Santiago-Bautista y Martínez-Domínguez (2017) para las revistas de Comunicación, Madrid et al. (2017) al analizar las revistas de educación, Reverter-Masía et al. (2014) en revistas de educación 
Bibliotecas. Vol. 39, №2, julio - diciembre, 2021. EISSN: 1659-3286

URL: http://www.revistas.una.ac.cr/index.php/bibliotecas/index

física y Crespo-Gascón, Tortosa y Guerrero-Casado (2019) en revistas de ciencias naturales.

Es claro que la ventaja de Brasil de poseer la mayor cantidad de revistas indexadas, también lo ubica como el país con la mayor cantidad de documentos publicados y citados, por lo que es más conveniente comparar con otras variables bibliométricas como el SJR y el número de citas por documento. Aun cuando el factor de impacto ha sido ampliamente estudiado y criticado en su esencia de cálculo, el que según investigadores como Garfield (2006) y Sugimoto (2016) está pensado para un mundo ideal donde los indicadores y revisores son infalibles, lo que evidentemente no es del todo cierto, en el caso de la comparación entre revistas es un buen indicador de diferenciación. En el caso particular, respecto al factor de impacto $(S J R)$ se observa que existe paridad en el impacto de las revistas de Argentina, Brasil y Chile a pesar de la diferencia en la cantidad de revistas y documentos publicados.

Las citas por documentos favorecen a las revistas de Chile, Ecuador y Colombia, por lo que se puede decir que para el año 2019 las revistas del área de ciencias ambientales que generaron mayor interés y por ende tuvieron mayor cantidad de citaciones de sus artículos publicados, fueran las de estos tres países. El número de citas por documentos muestra tendencia diferente al $S J R$, debido a que el primero es un indicador que se refiere al índice de inmediatez promedio de los artículos que se publican en una revista, es decir, no está en función de las citas en varios años, sino en las que recibe la revista en un año específico (De Vito, 2006) en este caso en 2019.

El análisis de los indicadores ambientales de los países con revistas de ciencias ambientales indexadas en Scopus, muestra que Chile y Brasil se distinguen de los demás países y se ubican ambos en clústeres independientes y además se diferencian entre ellos. En el caso chileno, en relación con el cuidado del ambiente tiene diversos matices, según Soto y Santibáñez (2003) en Chile los procesos de desertificación son evidentes, pero para enfrentarlos hay medidas más o menos conservacionista del medio, las cuales varían desde la completa indiferencia por parte de algunos que sobreutilizan los recursos buscando maximizar el beneficio a corto plazo, hasta conservacionistas extremos que no quieren que se obtenga provecho de los recursos, llegando incluso a negar el manejo sostenible de los mismos. En el caso de Brasil, la valoración del ambiente es un factor de importancia, según 
lo manifiestan Barbosa, Campanha, Oliveira y Gonçalves (2011) en el país se contempla el pago por servicios ambientales a los dueños de propiedades, que los imposibilita para explotar económicamente áreas de su terreno, las cuales están bajo protección para evitar que sean invadidas o degradadas.

Argentina y México se agrupan en un clúster, lo que indica que ambos países tienen indicadores ambientales similares. En el caso argentino, investigaciones como la de Zulaica y Tomadoni (2015) indican que en el país la situación de los indicadores ambientales es más crítica en los medios rurales, sobre todo por las precarias condiciones de vida, lo que coincide con lo expresado por Romanelli y Massone (2016) quienes indican que en Argentina se deben plantear y efectuar acciones que permitan una adecuada planificación y manejo de los recursos naturales. En el caso mexicano, también se observan coincidencias en cuanto a la importancia de los indicadores ambientales y se destaca que los mismos deben ser fundamentales para evaluar las condiciones del ambiente sobre todo en zonas rurales y susceptibles a intervención (Perevochtchikova, 2013).

Por otro lado, están Colombia, Ecuador y Cuba agrupados, lo que muestra a estos tres países con indicadores ambientales similares, pero que en conjunto son diferentes a los demás. En estos últimos tres países parece que lo común es una deficiente política en materia ambiental y que no existen fuentes confiables de indicadores, como lo mencionan en el caso de Colombia, González y Echeverry-Galvis (2019), en Ecuador, Carrión y Bravo (2015) y en el caso cubano Miranda, Betancourt y Santos (2018).

La relación entre las variables bibliométricas y los indicadores ambientales demostró que solo la cantidad de revistas científicas que posee cada país y el número de Autocitas muestran relación significativa con indicadores ambientales, siendo el común las Áreas protegidas. Lo anterior sugiere que mientras mayor cantidad de áreas protegidas tiene un país, es mayor la probabilidad de que tenga revistas en el área de ciencias ambientales indexadas en Scopus o WoS. Lo anterior contrasta con lo reportado por Crespo-Gascón et al. (2019) quienes concluyen que en Latinoamérica una mayor riqueza de recursos naturales no implica necesariamente una mayor producción de revistas científicas en ciencias naturales, lo que es evidente que no es el caso de las ciencias ambientales. En otros países de Latinoamérica como el caso del Perú, se han reportado relaciones directas entre las áreas 
protegidas y la investigación científica (Dourojeanni, 2018). Así mismo, Álvarez (2010) destacó la importancia de las áreas naturales protegidas en el desarrollo de la investigación básica y aplicada ya que estas son lugares idóneos para que se lleven a cabo programas de investigación.

Se observa de igual forma que en todas las relaciones significativas entre las variables bibliométricas y los indicadores ambientales, existe una relación inversa con los niveles de contaminación por material particulado (PM2.5) lo que sugiere que la tendencia es que los países latinoamericanos que poseen mayor cantidad de revistas científicas en ciencias ambientales presentan menor contaminación por PM2.5. En el caso de Brasil estudios como el de Blanco y Costa (2018) demostraron que la contaminación con PM2.5 es importante, ya que existe evidencia de relación con enfermedades respiratorias en el país, lo que es relevante por el hecho de ser este el país con mayor número de revistas en el área y con menor colaboración internacional comparado con otros países como Colombia y México, por lo que sus publicaciones tienden a ser de investigadores locales.

Igualmente, el resultado de los modelos matemáticos obtenidos también se contradice en el caso del país con la segunda mayor cantidad de revistas, el cual fue México, donde la contaminación atmosférica por material particulado es importante y se ha demostrado en diferentes investigaciones (Canales-Rodríguez et al., 2014; Legarreta et al., 2015; Sheinbaum, 2016). Lo anterior indica que, aunque se encontró relación significativa entre el número de revistas y las Autocitas con ciertos indicadores ambientales, se debe estudiar las mismas desde otras perspectivas y con otras variables, ya que la producción científica de los países va a depender de las políticas propias de los mismos y de los incentivos que se realicen para la protección del ambiente.

\section{v. Conclusiones}

Se concluye que en ALyC son pocas las revistas del área de ciencias ambientales indexadas en Scopus y WoS en comparación con otras áreas de conocimiento. Lo anterior limita la difusión del conocimiento y en cierta forma la investigación, a pesar de ser una región con gran biodiversidad y áreas naturales únicas que deben ser protegidas. Brasil, México y Colombia se ubican como los países con mayor número de revistas en el área. 
Existe relación estadísticamente significativa entre la cantidad de revistas latinoamericanas en el área de ciencias ambientales indexadas en Scopus y WoS y los indicadores ambientales Uso de Energía, Áreas protegidas, Contaminación por PM2.5 y las Rentas totales por recursos naturales. El indicador ambiental más influyente de forma positiva fue la cantidad de Áreas protegidas y de forma negativa influyen la Contaminación por PM2.5 y las Rentas totales por Recursos Naturales.

De las variables bibliométricas solo las Autocitas mostraron relación estadísticamente significativa con indicadores ambientales. Las Autocitas se relacionaron positivamente con las Áreas protegidas y negativamente con la Contaminación por PM2.5 y las Rentas por Recursos Naturales. El resto de las variables bibliométricas no mostraron relación significativa con los indicadores ambientales estudiados.

Se aconseja que se continúe investigando en esta misma línea y se incluyan variables que midan la promoción de la investigación por cada país del área, así como comparar los indicadores entre países con revistas indexadas en las bases de datos tomadas y los que no, para establecer una mejor relación entre estas variables y mostrar un panorama más general de las relaciones establecidas.

\section{Referencias}

Álvarez, K. (2010). Ciencia e investigación en las áreas protegidas. Aproximación al caso de la Reserva de la Biosfera de Urdaibai. Munibe (Ciencias Naturales-Natur Zientziak), 58, 233-248. Recuperado de: http://www.aranzadi.eus/fileadmin/docs/Munibe/2010233248CN.pdf

Amat, J. (2020). Comparación de distribuciones: test Kolmogorov-Smirnov. Recuperado de: https://tinyurl.com/yymxrg77

Barbosa, M., Campanha, A.C., Oliveira, M., Gonçalves, L.A. (2011). Criterios e indicadores para la valoración de los servicios ambientales en Brasil. Revista Letras Verdes, 9, 48-64. DOI: https://doi.org/10.17141/letrasverdes.9.2011.903

Blanco, A., Costa, L.F. (2018). Effects of exposure to air pollutants on children's health in Cuiabá, Mato Grosso State, Brazil. Caderno de Saúde Pública, 34(3), 1-9. DOI: https://doi.org/10.1590/0102-311x00006617

Canales-Rodríguez, M., Quintero-Núñez, M., Castro-Romero, T., García-Cuento, R. (2014). Las partículas respirables PM10 y su composición química en la zona urbana y rural de Mexicali, Baja California en México. Información Tecnológica, 25(6): 13-22. DOI: http://doi.org/10.4067/S0718-07642014000600003 
Carrión, D., Bravo, D. (2015). Sustentabilidad ambiental de los municipios: el caso de Ecuador. Fronteiras: Journal of Social, Technological and Environmental Science, 4(2), 115-131. DOI: http://doi.org/10.21664/2238-8869.2015v4i2.p115-131

Carvajal-Tapia, A.E., Carvajal-Rodríguez, E. (2019). Producción científica en ciencias de la salud en los países de América Latina, 2006-2015: análisis a partir de SciELO. Revista Interamericana de Bibliotecología Medellín (Colombia), 42(1), 15-21. DOI: https://doi.org/10.17533/udea.rib.v42n1a02

Casas, A., Torres, I., Delgado-Lemus, A., Rangel-Landa, S., Ilsley, C., Torres-Guevara, J., Cruz, A., et al. (2017). Ciencia para la sustentabilidad: investigación, educación y procesos participativos. Revista Mexicana de Biodiversidad, 88(1), 113-128. DOI: https://doi.org/10.1016/..rmb.2017.10.003

Celaya, A., Luque, D., García, J., Amozurrutia. J.A., Preciado, J.M., Laborín, J., Cabanillas, R.E. (2017). Evaluación de la producción científica de sustentabilidad ambiental en un centro público de investigación (CPI) del Conacyt (1982-2012). Revista de la Educación Superior, 46(182), 89-112. DOI: https://doi.org/10.1016/i.resu.2017.04.002

Crespo-Gascón, S., Tortosa, F., Guerrero-Casado, J. (2019). Producción de revistas científicas en América Latina y El Caribe en Scopus, Journal Citation Reports y Latindex en el área de los recursos naturales: su relación con variables económicas, ambientales y de inversión en investigación. Revista Española de Documentación Científica, 42(1), e224. DOI: https://doi.org/10.3989/redc.2019.1.1533

Cuadras, C. (2014). Nuevos métodos de análisis multivariante. Barcelona: CMC Editions, Recuperado de:

http://www.majefre.udc.es/wpcontent/uploads/2015/03/metodos multivariantes.pdf

De La Fuente, S. (2011). Análisis conglomerados. España: Universidad Autónoma de Madrid. Recuperado de: http://www.estadistica.net/MasterEconometria/Analisis Cluster.pdf

De Vito, E.L. (2006). Algunas consideraciones en torno al uso del Factor de Impacto y de la Bibliometría como herramienta de evaluación científica. Revista Americana de Medicina Respiratoria, 6(1), 37-45. Recuperado de: https://www.redalyc.org/pdf/3821/382138365005.pdf

Dourojeanni, M. (2018). Áreas naturales protegidas e investigación científica en el Perú. Revista Forestal del Perú, 33(2), 91-101. DOI: http://dx.doi.org/10.21704/rfp.v33i2.1223

Escalona, M.I., Lagar, P., Pulgarín, A. (2010). Web of Science vs. Scopus: un estudio cuantitativo en ingeniería química. Anales de Documentación, 13, 159-175. Recuperado de: https://revistas.um.es/analesdoc/article/view/107121

Garfield, E. (2006). The history and meaning of the journal impact factor. Journal of the American Medical Association, 295(1), 90-93. DOI: http://dx.doi.org/10.1001/jama.295.1.90

González, A.M., Echeverry-Galvis, M.Á. (2019). Indicadores ambientales y desempeño ambiental: Colombia en el índice de desempeño ambiental (EPI) (2006-2014). Ambiente y Desarrollo, 23(44), 1-18. DOI: https://doi.org/10.11144/Javeriana.ayd2344.iada 
Guerrero-Casado, J. (2017). Producción científica latinoamericana indexada en Scopus en el área de las ciencias agropecuarias: análisis del período 1996-2016. IDESIA (Chile), 37(4), 27-33. DOI: http://dx.doi.org/10.4067/S0718-34292017000400027

Ibáñez, J.J. (2017). La ciencia en Latinoamérica: tendencias y patrones. Revista de la Facultad de Ciencias, Universidad Nacional de Colombia, Sede Medellín, 7(1), 23-39. DOI: https://doi.org/10.15446/rev.fac.cienc.v7n1.69409

Legarreta, A., Corral, A.Y., Delgado, M., Torres, J., Flores, J.P. (2015). Material particulado y metales pesados en aire en ciudades mexicanas. CULCyT, 12(56), 234-245. Recuperado de: https://erevistas.uacj.mx/ojs/index.php/culcyt/article/view/818

León, J.L., Socorro, A.R., Cáceres, M.L., Pérez, J. (2020). Producción científica en América Latina y el Caribe en el período 1996-2019. Revista Cubana de Medicina Militar, 49(3), e0200573.

Recuperado

de:

http://www.revmedmilitar.sld.cu/index.php/mil/article/view/573

Luna-Solis, Y. (2015). Producción científica en salud mental en Perú. Reto en tiempos de reforma de salud. Acta Médica Peruana, 32(1), 36-40. DOI: https://doi.org/10.35663/amp.2015.321.171

Madrid, M.J., Jiménez-Fanjul, N., León-Mantero, C., Maz-Machado, A. (2017). Revistas brasileñas de Educación en SCOPUS: un análisis bibliométrico. Biblios, 67, 30-41. DOI: https://doi.org/10.5195/biblios.2017.344

Miguel, S. (2011). Revistas y producción científica de América Latina y el Caribe: su visibilidad en SciELO, RedALyC y SCOPUS. Revista Interamericana de Bibliotecología, 34(2), 187-199. Recuperado de: https://www.redalyc.org/articulo.oa?id=179022554006

Miranda, R.L.; Betancourt, Y., Santos, L. (2018). Indicadores de evaluación del desempeño ambiental en una organización cubana. Revista Ingeniería Industrial, 17(2), 149-170. DPI: https://doi.org/10.22320/S07179103/2018.09

Montilla, L.J. (2012). Análisis bibliométrico sobre la producción científica archivística en la Red de Revistas Científicas de América Latina y el Caribe (Redalyc) durante el período 2001-2011. Biblios, 48, 1-11. DPI: https://doi.org/10.5195/biblios.2012.65

Ortega-Loubon, C., Zúñiga-Cisneros, J., Yau, A., Castro, F., Barría-Castro, J.M., Lalyre, A., Silva, S., Rodríguez-Barría, E., Lezcano, H., Ortega-Paz, L. (2013). Producción científica de los estudiantes de medicina de la Universidad de Panamá. Archivos de medicina, 9(3), 1-9. Recuperado de: https://www.archivosdemedicina.com/medicinade-familia/produccin-cientfica-de-los-estudiantes-de-medicina-de-la-universidad-depanam.pdf

Perevochtchikova, M. (2013). La evaluación del impacto ambiental y la importancia de los indicadores ambientales. Gestión y Política Pública,12(2), 283-312. Recuperado de: http://www.scielo.org.mx/pdf/gpp/v22n2/v22n2a1.pdf

Reverter-Masía, J., Hernández-González, V., Jove-Deltell, C., Fonseca, T., Legaz-Arrese, A. (2014). Producción de artículos en la base de datos Web of Science y Scopus sobre educación física: estudio comparativo entre España y Brasil. TransInformação, Campinas, 26(2), 113-124. DOI: http://dx.doi.org/10.1590/0103-37862014000200001 
Rogel-Salazar, R., Santiago-Bautista, I., Martínez-Domínguez, N. (2017). Revistas científicas latinoamericanas de Comunicación indizadas en WoS, Scopus y bases de datos de Acceso Abierto. Comunicación y Sociedad, 30, 167-196. Recuperado de: http://www.scielo.org.mx/pdf/comso/n30/0188-252X-comso-30-00167.pdf

Romanelli, A., Massone, H.E. (2016). Desarrollo de indicadores ambientales e índice de calidad de lagos someros pampeanos de Argentina con alta intervención antrópica. Tecnología y Ciencias del Agua, 7(6), 123-137. Recuperado de: http://www.revistatyca.org.mx/index.php/tyca/article/view/1286

Santa, S., Herrero, V. (2010). Producción científica de América Latina y el Caribe: una aproximación a través de los datos de Scopus (1996-2007). Revista Interamericana de la Biblioteca, 33, 379-400. Recuperado de: https://revistas.udea.edu.co/index.php/RIB/article/view/7648

Sheinbaum, C. (2016). Contaminación atmosférica en la Zona Metropolitana del Valle de México. Recuperado de: https://tinyurl.com/y5j|75wb

Soto, G., Santibáñez, F. (2003). Uso de imágenes satelitales de baja resolución e indicadores ambientales, en el análisis de las relaciones entre la desertificación y la población en la región árida de Chile. En Livenais, P. y Aranda, X. (Eds.) Dinámicas de los sistemas agrarios en chile árido: la región de Coquimbo (p. 255-270). Marsella: IRD Éditions. Recuperado de: https://books.openedition.org/irdeditions/24953?lang=es

Statgraphics Centurion XVII (Versión 17.2.07; Statgraphics Technologies, Inc: 2018). https://www.statgraphics.com/download-statgraphics-centurion-xvii

Sugimoto, C.R. (2016). Theories of informetrics and scholarly communication. Berlín: De Gruyter. DOI: https://doi.org/10.1515/9783110308464

Zulaica, L., Tomadoni, M. (2015). Indicadores de sostenibilidad ambiental en el periurbano de la Ciudad de Mar del Plata, Argentina. Anales de Geografía, 35(2), 195-216. DOI: https://doi.org/10.5209/rev AGUC.2015.v35.n2.50120

\section{Notas de las autorías}

Tomás Darío Marín Velásquez. Magíster en Ingeniería y Tecnología Ambiental. Universidad de Oriente, Venezuela. Correo electrónico tmarin@protonmail.com. ORCID: https://orcid.org/0000-0002-3334-5895

Dany Day Josefina Arriojas Tocuyo. MBA en Gerencia de la industria de Hidrocarburos. Petróleos de Venezuela. Correo electrónico arriojasd@gmail.com. ORCID: https://orcid.org/0000-0002-8192-9641 